\title{
Ultramicroscopy: Light-Sheet-Based Microscopy for Imaging Centimeter-Sized Objects with Micrometer Resolution
}

\author{
Klaus Becker, Nina Jährling, Saiedeh Saghafi, and Hans-Ulrich Dodt
}

\begin{abstract}
Ultramicroscopy (UM) is a powerful imaging technique that achieves precise and accurate threedimensional (3D) reconstructions of intact macroscopic specimens with micrometer resolution. It was developed for specimens in the size range of $\sim 1-15 \mathrm{~mm}$, such as whole mouse brains, mouse embryos, mouse organs, and Drosophila melanogaster. In UM, the specimen is illuminated perpendicular to the observation pathway by two thin counterpropagating sheets of laser light. UM is closely related to a growing family of comparable microscopy approaches based on light sheet illumination developed in recent years. This article presents an overview of light-sheet-based microscopy and describes the underlying physics of light sheet generation. The assembly of an "ultramicroscope" for investigating fixed chemically cleared tissue is described in detail, and the functions of the essential components, such as mechanics, camera, and objectives, are discussed. Finally, practical applications of UM for studying mouse organs, mouse embryos, and Drosophila adults are described.
\end{abstract}

Detailed three-dimensional (3D) anatomical reconstruction of intact specimens (e.g., whole mouse brains and embryos), which range in size from several millimeters to centimeters, is extremely difficult using conventional light microscopy. Alternatives such as micromagnetic resonance imaging ( $\mu \mathrm{MRI})$ are currently not capable of achieving the required resolution (Tyszka et al. 2005) and cannot be combined with fluorescence labeling (Tyszka et al. 2005). Consequently, until quite recently, techniques involving mechanical slicing procedures, despite being labor intensive, were the only methods available for overcoming these problems, although the results obtained were often unsatisfactory. Some problems, such as mechanical tissue distortion by the microtome knife, are unavoidable, and the complexity of reference point mapping associated with accurately and precisely registering hundreds of single sections to create a reliable 3D model force the investigator to compromise on the desired results (Weninger and Mohun 2002).

Ultramicroscopy (UM) is a microscopy technique in which the specimen is illuminated perpendicular to the observation pathway by two thin counterpropagating sheets of laser light, which are formed by one or more optical components (e.g., cylindrical lenses; Fig. 1). Thus, only the in-focus "area" of the specimen is illuminated. Hence, out-of-focus "areas" cannot contribute to stray-light generation and image blurring. The two-sided illumination is especially useful for large specimens, such as whole mouse brains, because it counterbalances the loss of light intensity caused by light absorption by the tissue to a certain degree. To further minimize the generation of stray light, typically specimens are chemically cleared before investigation.

$\mathrm{UM}$ achieves precise and accurate 3D reconstructions of macroscopic specimens with micrometer resolution, without requiring microtome slicing (Dodt et al. 2007; Becker et al. 2008; Jährling et al.

Adapted from Imaging: A Laboratory Manual (ed. Yuste). CSHL Press, Cold Spring Harbor, NY, USA, 2010.

(C) 2013 Cold Spring Harbor Laboratory Press

Cite this article as Cold Spring Harb Protoc; 2013; doi:10.1101/pdb.top076539 


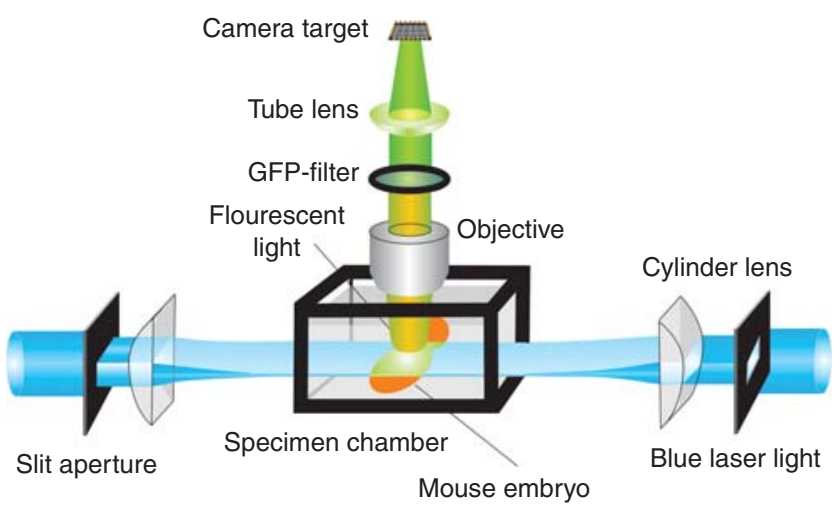

FIGURE 1. Principle of UM. The sample is illuminated from the side by two thin counterpropagating sheets of laser light. Fluorescent light is thus emitted only from a thin optical section and collected by the objective. Hence, photobleaching in out-of-focus regions is avoided. (Adapted, with permission, from Becker et al. 2008.)

2008). UM is closely related to a growing family of comparable microscopy approaches based on light sheet illumination developed in recent years. Among these techniques are single-plane illumination microscopy (SPIM) (Huisken et al. 2004), orthogonal-plane fluorescence optical sectioning (OPFOS) (Voie et al. 1993), and a number of other methods utilizing the same principle (Huisken and Stainier 2009). Light sheet illumination has been used for more than a century and can be traced to an invention by Siedentopf and Zsigmondy (1903), in which the term ultramicroscopy was originally coined. Using UM, it is possible to generate a three-dimensional reconstruction of a whole mouse embryo in $<1 \mathrm{~h}$. The technique can be applied to immunolabeled specimens, XFP-expressing transgenic animals, and autofluorescence investigations. Speed and simplicity make UM ideally suited for high-throughput phenotype screening of transgenic mutants of mice, flies, and the large number of other specimens generated by biomedical research.

\section{PRINCIPLE OF ULTRAMICROSCOPY}

Imaging intact macroscopic specimens requires large fields of view, which necessitates the use of objectives with a low numerical aperture (NA). Unfortunately, these normally have poor axial resolution, thus restricting $3 \mathrm{D}$ reconstructions using either confocal or two-photon microscopy to specimen sizes that are less than a few hundred micrometers (Helmchen and Denk 2005). Efforts have been made to overcome this limitation using computational techniques such as wide-field deconvolution (Shaw 1995). Although such an approach has become more applicable with increases in computing power and memory, it still can be applied in real time. Furthermore, the results are severely limited by the presence of noise (Shaw 1995). In UM, the illumination and the observation pathway of the microscope are separated to achieve an uncoupling of lateral and axial resolution. One consequence is that the axial resolution is not limited by the NA of the objective, but by the thickness of the light sheet. In UM, optical sectioning is achieved by stepping the specimen chamber vertically through the light sheet in small increments using a computer-controlled jack.

Using light sheet illumination, no photobleaching is generated in the out-of-focus planes of the specimen, because these are always in the dark. Critically, when working with highly bleachable fluorophores, this is an important advantage compared with standard confocal microscopy.

Theory of Light Sheet Generation by a Single Cylinder Lens

The spot size of a symmetrical ideal Gaussian beam along the propagation axis is generally given by 
K. Becker et al.

$$
w(z)=w_{0} \sqrt{1+\left(\frac{\lambda \cdot z}{\pi \cdot w_{0}^{2}}\right)^{2}} .
$$

In practice, most lasers do not produce diffraction-limited Gaussian beams. The output intensity distribution of a real laser beam is related to an ideal Gaussian beam (TEM ${ }_{00}$ mode) through a standard parameter (ISO Standard 11146), the $M^{2}$-beam propagation factor (Siegman 1998). $M^{2}$ is usually provided by the laser manufacturer; if not, there are several standard methods for measuring this factor experimentally (ISO Standard 11146; Sasnett and Johnston 1991). Considering $M^{2}$, Equation 1 becomes, for the $x$ direction,

$$
w_{x}^{2}=w_{0 x}^{2}+M_{x}^{4} \times\left(\frac{\lambda}{\pi w_{0 x}}\right)^{2}\left(z-z_{0 x}\right)^{2}
$$

and, similarly for the $y$ direction,

$$
w_{y}^{2}=w_{0 y}^{2}+M_{y}^{4} \times\left(\frac{\lambda}{\pi w_{0 y}}\right)^{2}\left(z-z_{0 y}\right)^{2}
$$

(Siegman 1998).

If a cylindrical lens of focal length $f$ is illuminated by a Gaussian beam, the beam width and curvature change in one direction, while both remain approximately unchanged in the direction perpendicular to the focused direction (Siegman 1986) (Fig. 2A).

The time-averaged intensity distribution of this beam of wavelength $\lambda$ along the $z$-axis can be described by

$$
I(r, z)=I_{0}\left(\frac{w_{0 x} w_{0 y}}{w_{x}(z) w_{y}(z)}\right) \exp \left(-2\left(\frac{x^{2}}{w_{x}^{2}(z)}+\frac{y^{2}}{w_{x}^{2}(z)}\right)\right)
$$

(Fig. 2A). In Equation 4, $x$ and $y$ are the distances from the center of the beam in Cartesian coordinates. $\omega_{0 x}$ and $\omega_{0 y}$ are the beam waist (minimum spot size value along the $z$-axis) at $x$ and $y$ directions, respectively. Similarly, $\omega_{x}(z)$ and $\omega_{y}(z)$ are the beam radius along the propagation axis $z$ at any arbitrary point (e.g., at $1 / \mathrm{e}^{2}$ cutoff values), and $I_{0}$ is the maximum intensity at the beam center. When the laser beam is focused, a new beam waist (constructed waist) is produced (Fig. 2A). The minimal half-width $\omega_{0 f}$ of this new constructed waist near the focal plane approximately is

$$
w_{0 f} \cong M^{2} \frac{\lambda \cdot f}{\pi \cdot w_{D}} .
$$

In Equation $5, \omega_{D}$ is the half-width of the lens-illuminating laser beam in the focused direction. The beam width and intensity, at any arbitrary point along the $z$ direction, can be obtained using Equations $2-5$ by replacing the beam waist with the constructed waist in the related direction.

\section{Effects of the Medium in the Specimen Chamber on the Beam Propagation}

The width $\omega_{D}$ of the beam illuminating the cylindrical lenses is controlled by removable slit apertures, which are placed directly before the cylindrical lenses (Fig. 1). Using different slit apertures (e.g., 2, 4, 6,8 , or $10 \mathrm{~mm}$ with cylindrical lenses of focal length $f=80 \mathrm{~mm}$ ) the shape of the light sheet can be adjusted.

As the illumination beam is refracted at the wall of the specimen chamber, owing to the changing refractive index, the numerical values $d$ for the width of the slit apertures have to be corrected by a 
A

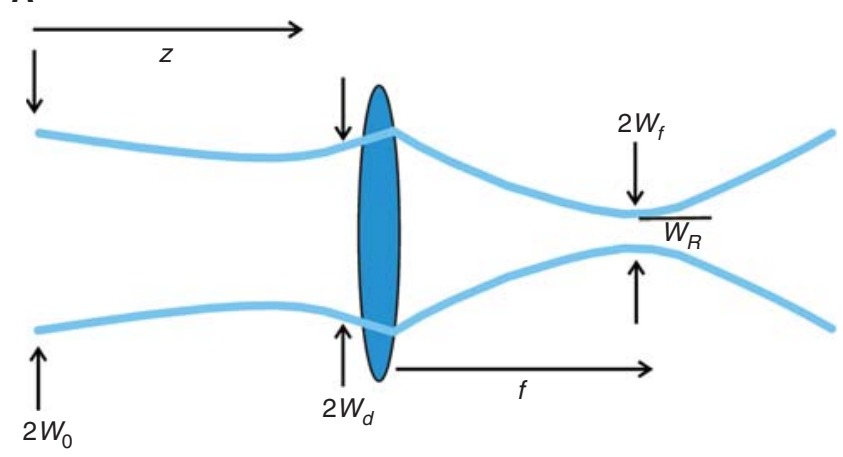

B

Slit aperture Cylindric lens

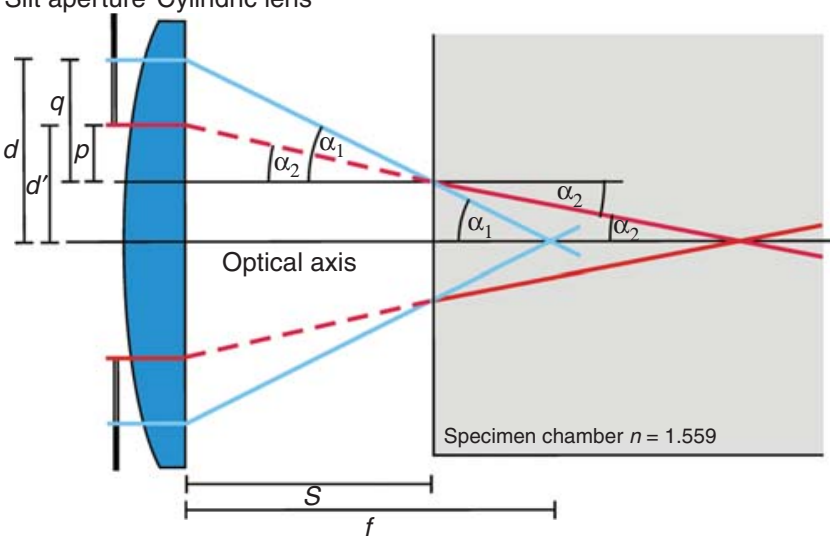

FIGURE 2. (A) Refraction of a real Gaussian beam $\left(M^{2}>1\right)$ at a single cylindrical lens. When the laser beam is focused, a new waist of the laser beam (constructed waist) is produced. Symbols: $z$, propagation axis; $\omega_{0}$, initial beam radius; $\omega_{\mathrm{D}}$, half-width of the lens-illuminating laser beam; $\omega_{\mathrm{f}}$, constructed beam waist; $\omega_{\mathrm{R}}$, Raleigh range. $(B)$ Because of the refraction at the specimen chamber, the effective width of the slit aperture $d^{\prime}$ is smaller than the width of the physical slit aperture $d$ by a certain factor $\gamma$. This factor depends on the distance between cylindrical lens and specimen chamber, the focal length of the cylindrical lens, and on the refractive index of the medium in the specimen chamber. (B, Modified, with permission, from Jährling et al. 2008, CElsevier GmbH.)

constant arbitrary number $\gamma$, because the effective slit aperture is smaller than the physical aperture $d$ (Fig. 2B):

$$
d^{\prime}=\gamma \cdot d
$$

$\gamma$ can be approximately determined from geometrical optics:

$$
\gamma=1-\frac{s}{f}+\frac{s}{\sqrt{n^{2}\left(f^{2}+d^{2}\right)-d^{2}}}
$$

(Jährling et al. 2008).

Figure 3 shows a plot of the simulated beam shapes calculated according to Equations 4-7 for different slit apertures ranging from $1 \mathrm{~mm}$ up to $10 \mathrm{~mm}$ width. Clearly, the waist $w_{f}$ of the light sheet becomes thinner with increasing slit widths; conversely, the Raleigh range $z_{\mathrm{R}}$ increases (Fig. $2 \mathrm{~A}$ ). Consequently, the width of the light sheet has to be individually modified for each specimen, depending on its size. 
K. Becker et al.

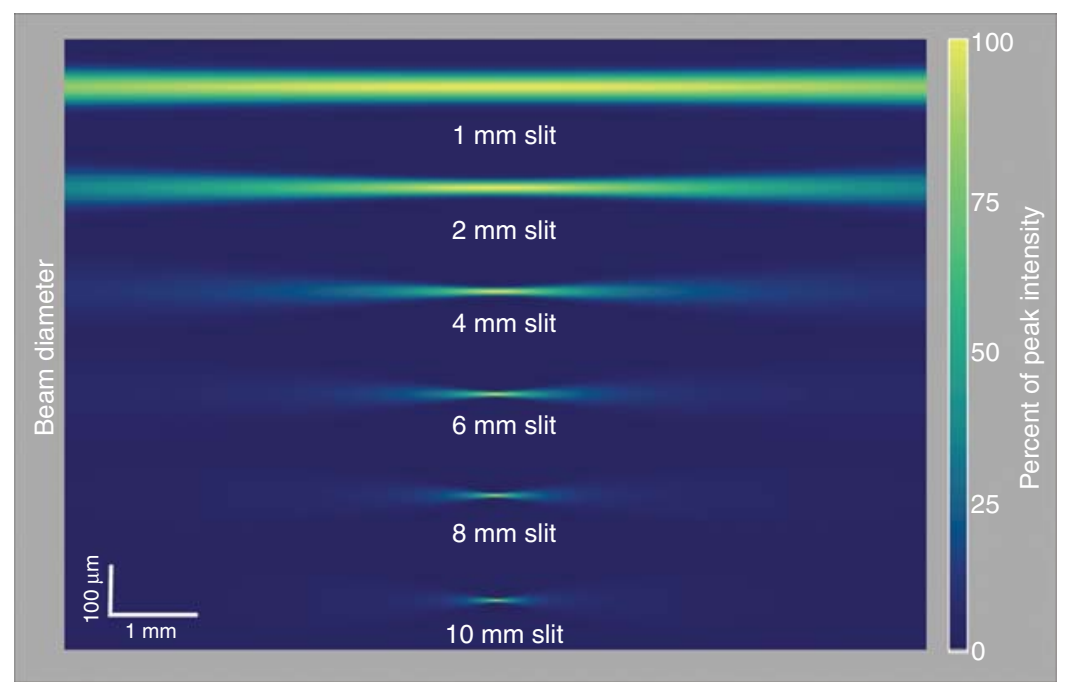

FIGURE 3. Spatial light intensity profiles for different values of the slit aperture width $d$, calculated according to Equations 4-7. The waist of the light sheet becomes thinner with increasing slit widths, but it strongly expands with increasing distance from the focus (simulation parameters: laser wavelength $\lambda=488 \mathrm{~nm}$, focal length of cylindrical lens $f=80 \mathrm{~mm}$, distance between cylindrical lens and specimen chamber $s=60 \mathrm{~mm}$, refractive index of specimen chamber medium $n=1.559$ ). (Modified, with permission, from Jährling et al. 2008, CElsevier $\mathrm{GmbH}$.)

\section{Specimen Dehydration and Clearing}

Because UM requires the excitation light sheet to travel throughout the entire horizontal width of the specimen, specimens usually have to be rendered transparent before microscope inspection. Such clearing can be obtained by a technique first applied by the German anatomist W. Spalteholz in his studies of heart vascularization (Spalteholz 1914). It relies on incubation of the specimen in an oily medium having almost the same refractive index as protein. Thus, light scattering is strongly reduced, and light traverses the specimen with minimal diffraction. Additionally, if the light absorption by dyes is low, the specimens will become almost completely transparent (Fig. 4). Detailed clearing procedures depend on the type and size of the specimen. Protocols have been established for Drosophila (see Dehydration and Clearing of Adult Drosophila for Ultramicroscopy [Becker at al. 2013a]), mouse embryos (see Immunostaining, Dehydration, and Clearing of Mouse Embryos for Ultramicroscopy [Becker et al. 2013b]), mouse brains, and isolated mouse hippocampi (see Dehydration and Clearing of Whole Mouse Brains and Dissected Hippocampi for Ultramicroscopy [Becker at al. 2013c]).

\section{Image Enhancement by Deconvolution}

The quality of ultramicroscopic 3D reconstructions can be significantly improved by deconvolution (Fig. 5). Commercial software packages like Huygens (Scientific Volume Imaging) or AutoQuant (Media Cybernetics) developed for confocal microscopy have been found suitable for this purpose. With Huygens the best results are obtained in the "confocal deconvolution" mode, using an experimentally determined point-spread function (PSF) (Jährling et al. 2008). Figure 5C shows a reconstruction obtained from mouse nerve fibers in the hindlimb of an E12.5 mouse. Figure 5D, obtained from the same stack of data, has been deconvolved using Huygens. As a result, fine details, like small fiber branches, become more pronounced and background intensity is strongly reduced (Fig. 5D).

Measuring the PSF of an UM setup can be performed with fluorescent beads that are $0.5-1 \mu \mathrm{m}$ in diameter (e.g., FluoSpheres, $1 \mu \mathrm{m}, 488 \mathrm{~nm}$; Invitrogen). Because the beads are delivered in an aqueous solution, which is not miscible with the solution in the specimen chamber, $1 \mu \mathrm{L}$ of the bead suspension is dried overnight in an open tube in a desiccator, and then mixed with $1 \mu \mathrm{L}$ of polyester resin (Polyester-Klarharz, Carl Roth GmbH, Germany), and $20 \mu \mathrm{L}$ of the included accelerator. Curing is performed overnight at room temperature in a small cube-shaped rubber form of $\sim 1 \times 1 \times 1$-cm feed 


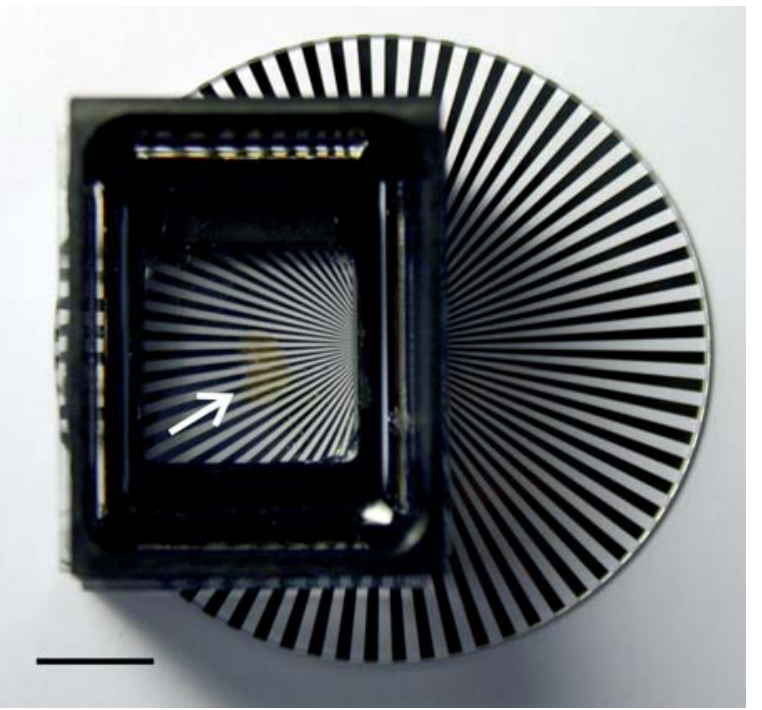

FIGURE 4. A mouse embryo E12.5 (light brown) placed in the specimen chamber filled with clearing solution (BABB). A Siemens star allows for an estimation of the degree of transparency. (Modified, with permission, from Jährling et al. 2008, (OElsevier GmbH.)

size. The cured resin has approximately the same refractive index as BABB $(n \approx 1.565)$. A stack of 150 200 images is recorded from the resin-embedded beads (Fig. 5A). To obtain sufficient resolution, either a twofold or fourfold postmagnification can be used. From the obtained image stack, a 3D model of the PSF can be calculated (e.g., by the "bead distiller" provided with Huygens; Fig. 5B). The PSF measurements have to be performed separately for different combinations of objectives and slit apertures. Before starting a certain deconvolution, the appropriate PSF data have to be loaded into
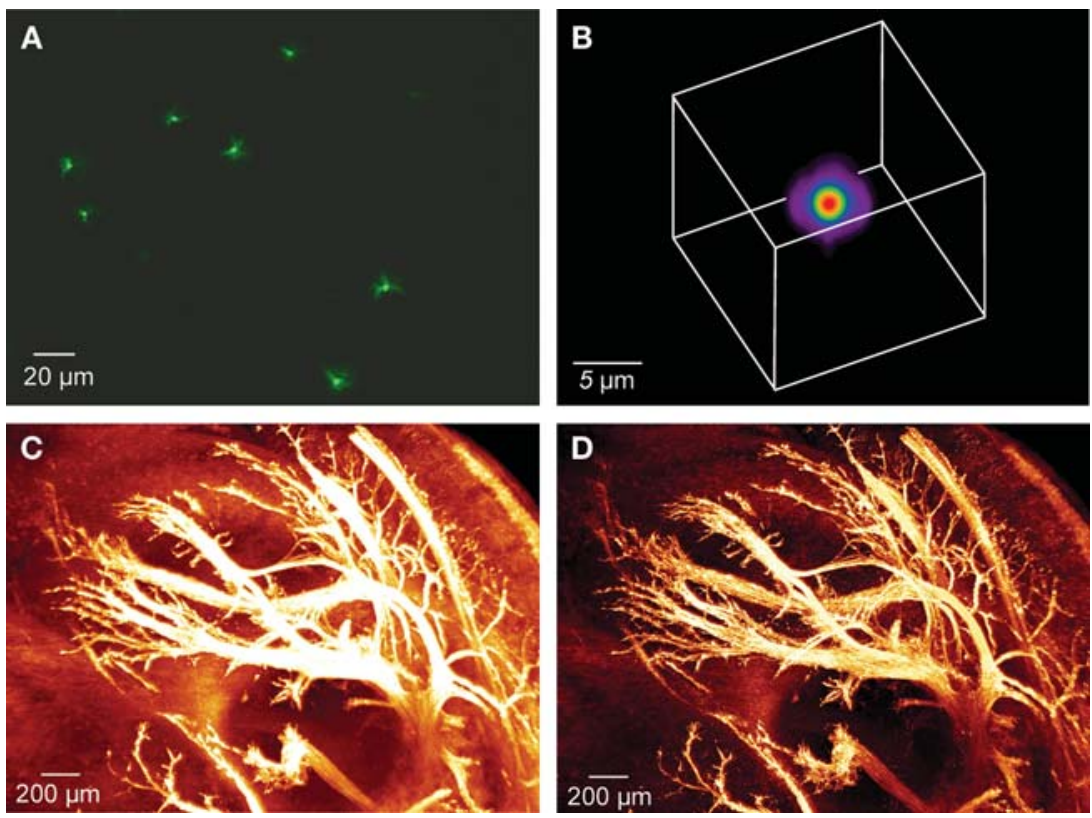

FIGURE 5. Quality improvement of UM reconstructions by nonblind deconvolution. (A) Resin-embedded latex beads for measuring the point-spread function (FluoSpheres $1 \mu \mathrm{m}, 488 \mathrm{~nm}$; Invitrogen). Objective: Olympus Fluar 10×, NA 0.3 with $4 \times$ postmagnification. $(B)$ PSF determined by averaging the bead recordings shown in $A$. (C) 3 D reconstruction of a part of the right hindlimb of a mouse embryo E12.5. (D) Deconvolution of $C$ using a measured PSF. In contrast to $C$ even fine nerve branches are distinguishable. (C,D, Reprinted, with permission, from Jährling et al. 2008, $O$ Elsevier $\mathrm{GmbH}$.) 
K. Becker et al.

Huygens. Because the deconvolution process is computationally very intensive and consumes an extensive amount of memory, the computer should be sufficiently powerful. For deconvolving overnight a large stack containing $\sim 2048 \times 2048 \times 800$ voxels, a multiprocessor system (e.g., two Intel Quad Core processors) equipped with at least $24 \mathrm{~GB}$ of main memory is recommended.

\section{ULTRAMICROSCOPY SETUP}

A complete ultramicroscope can be configured at relatively low cost using standard components from various optics and microscope distributors. To avoid artifacts from mechanical vibrations, the whole UM setup should be assembled on a vibration-cushioned optical breadboard (e.g., $150 \times 90 \mathrm{~cm}$ in size) (Fig. 6).

\section{Mechanical Components}

The microscope stand (e.g., Leica), the jack for vertically moving the specimen chamber, and two linear stages (e.g., miCos $\mathrm{GmbH}$ ) for fine focus adjustment of the cylindrical lenses are the essential mechanical components of an UM setup. These components are operated by computer-controlled stepping motors, allowing precise and vibration-free finetuning in the submicrometer range. In our laboratory, the optical components for light sheet generation, like lenses and mirrors, are assembled using the "microbench" optical construction system from LINOS Photonics. Some minor elements (e.g., the holders for the $45^{\circ}$ tilted mirrors) are custom-made (Fig. 6).

\section{$\stackrel{\infty}{\circ}$ Optical Components}

The microscope consists of a binocular eyepiece (e.g., Olympus) with integrated camera interface, mounted on a custom-made intermediate tube equipped with a thread at its bottom side for aligning the objectives. The tube fits into the holding ring of the microscope stand and has a collateral slit for inserting a variety of optical detection filters.

For projecting the microscopic image onto the camera target, we use standard fluorescence objectives (XL Fluor $2 \times$ NA 0.14, XL Fluor $4 \times$ NA 0.28 , or Fluor $10 \times$ NA 0.30; Olympus). The objectives are encased by custom-made plastic caps, having an $\sim 0.5$-mm-thick circular glass window at their lower surface. The caps allow for the immersion of the objective into the specimen chamber medium without damage. As the objectives are calculated for air, image quality suffers from

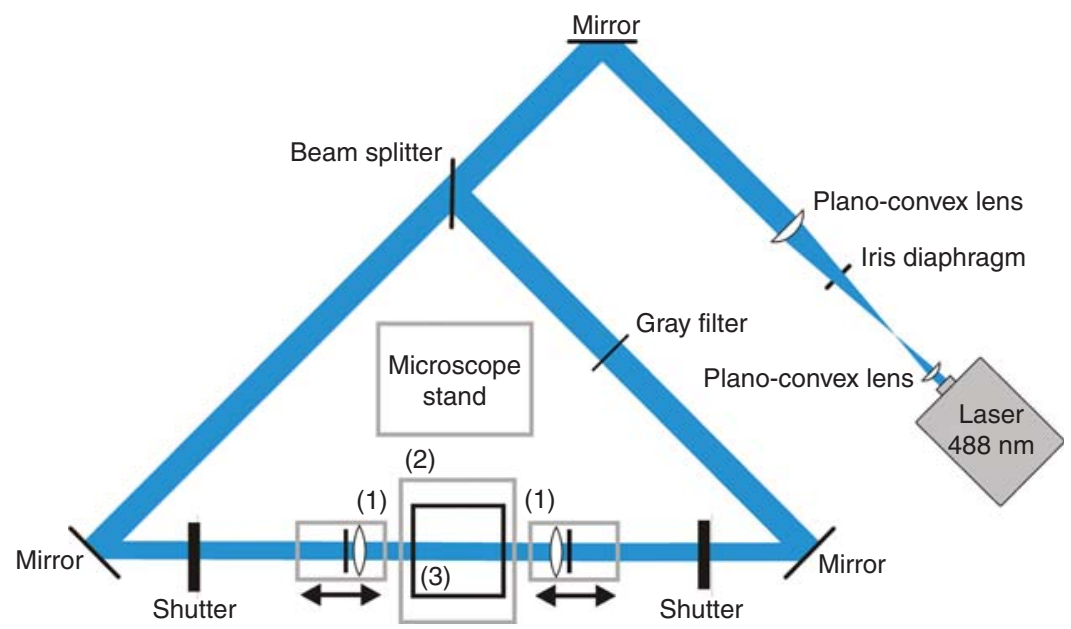

FIGURE 6. Schematic drawing of the UM setup. (1) Motor-driven linear stages with cylindrical lenses and slit apertures. (2) Computer-controlled jack. (3) Specimen chamber filled with clearing medium. (Modified, with permission, from Jährling et al. 2010.) 
a certain degree of spherical aberration, when viewing specimens embedded in a highly refractive medium, such as BABB. The caps ensure that the optical pathway through the medium between the light sheet and the front lens of the objective always remains constant, while stepping the specimen chamber vertically through the light sheet. Thus, the degree of spherical aberration does not depend on how deeply one focuses into the specimen.

As a light source, we use a 488-nm optically pumped semiconductor laser of $500 \mathrm{~mW}$ power (sapphire; Coherent), generating a monomode laser beam with an approximately Gaussian intensity profile $\left(\mathrm{TEM}_{00}, M^{2}=1.1\right)$, and a beam diameter of $0.7 \pm 0.05 \mathrm{~mm}$. The beam is expanded and homogenized by two convex lenses of 6-mm and 200 - $\mathrm{mm}$ focal length to a diameter of $\sim 15 \mathrm{~mm}$ (Fig. 6).

A system of mirrors and a beam splitter is used to guide the laser beam into two cylindrical lenses of $80-\mathrm{mm}$ focal length, placed at the left and right side of the specimen chamber. Custom-made holders are mounted directly behind the lenses, which allow for the insertion of different slit apertures, ranging from 2 to $16 \mathrm{~mm}$ in width. As discussed before, these slit apertures aid in shaping the light sheet to accommodate different specimen sizes (Fig. 6).

\section{Camera and Software}

For data sampling, we use a CoolSNAP K4 cooled charge-coupled device (CCD) camera with $15.16 \times$ 15.16-mm target size $(2048 \times 2048$ pixels $)$ (Roper Scientific). In general, low-power objectives (e.g., $2 \times-20 \times$ magnification) are used in UM. Consequently, when selecting a camera, possible limitations of resolution owing to the size of the camera target have to be taken into consideration. Using a $4 \times$ objective with a NA of, for example, 0.28 , the theoretical lateral resolution of the objective according to Raleigh's criterion is $\sim 1.1 \mu \mathrm{m}(\lambda=510 \mathrm{~nm})$. On the other hand, the back-projected pixel size in the object plane would be only $15.16 \mathrm{~mm} /(2048 \times 4)=1.85 \mu \mathrm{m}$, in this example, without using any postmagnification. According to Nyquist's theorem, at least $2 \times 2$ pixels (more, in practice) are needed to resolve a given detail; and a back-projected pixel size of $(1.1 / 2) \times(1.1 / 2) \mu \mathrm{m}$ would be required for fully using the resolving power of the objective. This results in an undersampling by a factor of $1.85 \mu \mathrm{m} / 0.55$ $\mu \mathrm{m}=3.4$. The limitations of today's scientific grade CCD cameras and computer hardware are such that undersampling cannot be completely avoided when working with low-power objectives. As a rule, to achieve the best sampling rates, the number of camera pixels should be as high as possible and the CCD sensor size should be small. Camera operation, jack, shutters, and the two linear stages are controlled by custom-made software programmed in Visual Studio 2008 (Microsoft), allowing for the automatic recording of an image stack once all optical components are correctly adjusted.

\section{PRACTICAL APPLICATION}

UM was developed for specimens in the size range of $\sim 1-15 \mathrm{~mm}$, such as whole mouse brains, mouse embryos, mouse organs, and Drosophila melanogaster. Specimens for UM need to be sufficiently transparent. This requires chemical clearing in most cases. Therefore, the specimens have to be able to withstand dehydration and treatment with chemical clearing agents. When working with XFPexpressing or immunolabeled material, the presence of autofluorescence can be a problem. Therefore, blood contained in mouse brains or organs has to be removed by careful transcardical perfusion before fixation. Specimens should not contain structures that strongly absorb light. In certain cases, pigments can be removed by bleaching with hydrogen peroxide (Dent et al. 1992). UM allows for discriminating between XFP-expressing neuronal somata and dendrites in the hippocampus through the overlying neocortex. In excised mouse hippocampi, dendritic networks of pyramidal neurons, up to the level of single spines, can be visualized (Dodt et al. 2007).

Imaging Autofluorescence

The simplest UM reconstruction technique is autofluorescence imaging. Because of the presence of endogenous chromophores, such as elastin, collagen, tryptophan, NADH, porphyrins, and flavins, 
K. Becker et al.
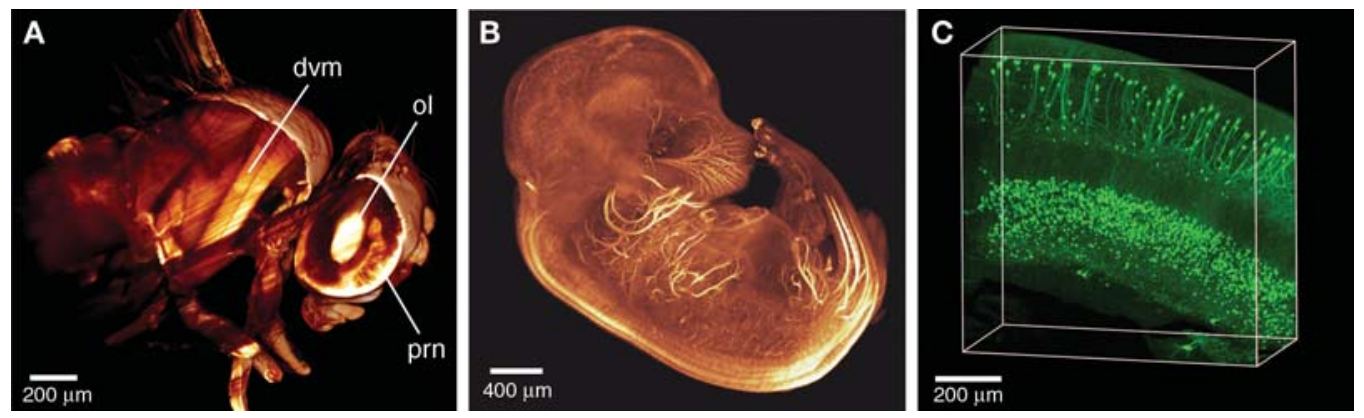

FIGURE 7. (A) View into the body of a Drosophila male, reconstructed from 238 optical sections. Easily recognized are some dorsoventral indirect flight muscles (ligamentous structures in the thorax, dvm), and one of the optic lobes as a part of the insect brain. $(B)$ Images obtained from a mouse embryo E12.5 immunostained with neurofilament 160, reconstructed from $4 \times 413$ optical sections. The thinnest sensory nerve fibers innervating the vibrissae are detectable. (C) 3D reconstruction of a part of a mouse hippocampus using 132 optical sections. The complex dendritic network of pyramidal cells becomes apparent in the reconstruction. (A, B, Reprinted, with permission, from Becker et al. 2008. C, Modified, with permission, from Dodt et al. 2007.)

with absorption spectra ranging from blue to ultraviolet, most biological tissues show a distinct autofluorescence throughout the visible to the near-infrared (Billinton and Knight 2001). Using a $488-\mathrm{nm}$ laser for excitation in combination with a $520 \mathrm{~nm} \pm 50 \mathrm{~nm}$ detection filter, the exterior and interior anatomy of Drosophila can be clearly imaged (Becker et al. 2008). This includes the direct and indirect flight muscles and structures of the nervous system, such as the optic lobes and the thoracic ganglia (Fig. 7A). Major blood vessels in mouse embryos and fiber tracts and the barrel cortex in isolated mouse brains can also be directly imaged using autofluorescence (Dodt et al. 2007).

\section{Immunostaining Mouse Embryos}

In combination with fluorescein isothiocyanate (FITC) immunostaining, UM allows visualization of somatic motor and sensorial nerve fibers in whole mouse embryos. Even the fine branches of the sensomotoric fibers can be visualized over a distance of up to several millimeters (Figs. 5C,D, and 7B) (Becker et al. 2008; Jährling et al. 2008). An immunostaining protocol is available (Immunostaining, Dehydration, and Clearing of Mouse Embryos for Ultramicroscopy [Becker et al. 2013b]).

\section{Lectin-Labeled Mouse Organs}

The vascular system can be labeled by infiltration of $\sim 10 \mathrm{~mL}$ of fluorescent conjugated lectins during transcardial perfusion (e.g., $10 \mu \mathrm{g}$ lectin-FITC from Lycopersicon esculentum per mL [L0401, SigmaAldrich]). This allows studies of vascular networks in mouse organs, like brains, spinal cord, or heart (Jährling et al. 2009).

\section{XFP-Expressing Transgenic Mice}

Green fluorescent protein (GFP)-labeled neurons can be imaged by UM in isolated hippocampi, as well as in whole brains of Thy-1 GFP-M (c57/Bl6) mice (Feng et al. 2000). The complex dendritic network of pyramidal neurons can be imaged using a $5 \times$ objective with NA 0.25 (Fig. 7C). By using a $20 \times$ objective providing a NA of 0.4 and deconvolution, spines can be identified in isolated mouse hippocampi (Dodt et al. 2007). For XFP-expressing specimens, it is essential that fluorescence be maintained during the clearing procedure. Thus clearing time should be kept as short as possible (e.g., $6 \mathrm{~h}$ for isolated mouse hippocampi and $\sim 2 \mathrm{~d}$ for whole mouse brains). 


\section{CONCLUSION AND FUTURE DIRECTIONS}

Ultramicroscopy has proven to be a powerful tool for the study of specimens that are too large to be imaged in a single pass by confocal microscopy but are too small to be accessible to macrophotography. Thus, UM bridges a gap in which previously no adequate imaging techniques were available at the required resolution. UM is capable of providing micrometer-level resolution in specimens that are in the centimeter size range. This opens up numerous novel applications, especially in neuroscience and developmental studies.

UM provides potential for further advances. Currently, commercial CCD cameras do not provide enough resolution to fully take advantage of low-power objectives with high numerical apertures. Furthermore, 3D reconstructions of very highly resolved image stacks are presently limited by available computing power. Some specimens show a distinct autofluorescence, which reduces the visualization depth for small details. To improve imaging depth, XFP fluorescence could be distinguished from autofluorescence by spectral imaging (Dickinson et al. 2001). Because low-power objectives corrected for use in oil are currently not commercially available, correction of spherical aberration by custom-made correction optics may improve resolution and imaging depth. By using more sophisticated optics for light sheet generation, thinner and more homogenous light sheets may be obtained. With further improvements, a UM-based microscope providing a resolution of $1 \mu \mathrm{m}$ throughout should make it possible to generate a whole mouse brain $3 \mathrm{D}$ reconstruction.

\section{REFERENCES}

Becker K, Jährling N, Kramer ER, Schnorrer F, Dodt HU. 2008. Ultramicroscopy: 3D-reconstruction of large microscopic specimens. J Biophotonics 1: $36-42$.

Becker K, Jährling N, Saghafi S, Dodt HU. 2013a. Dehydration and clearing of adult Drosophila for ultramicroscopy. Cold Spring Harb Protoc doi: 10.1101/pdb.prot075812.

Becker K, Jährling N, Saghafi S, Dodt HU. 2013b. Immunostaining, dehydration, and clearing of mouse embryos for ultramicroscopy. Cold Spring Harb Protoc doi: 10.1101/pdb.prot076521.

Becker K, Jährling N, Saghafi S, Dodt HU. 2013c. Dehydration and clearing of whole mouse brains and dissected hippocampi for ultramicroscopy. Cold Spring Harb Protoc doi: 10.1101/pdb.prot075820.

Billinton N, Knight A. 2001. Seeing the wood through the trees: A review of techniques for distinguishing green fluorescent protein from endogenous autofluorescence. Anal Biochem 291: 175-197.

Dent JA, Cary RB, Bachant JB, Domingo A, Klymkowski MW. 1992. Host cell factors controlling vimentin organization in the Xenopus oocyte. $J$ Cell Biol 119: 855-866.

Dickinson ME, Bearman G, Tille S, Lansoford R, Fraser SE. 2001. Multispectral imaging and linear unmixing add a whole new dimension to laser scanning fluorescence microscopy. BioTechniques 31: 12721278.

Dodt HU, Leischner U, Schierloh A, Jährling N, Mauch CP, Deininger K, Deussing JM, Eder M, Zieglgänsberger W, Becker K. 2007. Ultramicroscopy: Three-dimensional visualization of neural networks in the whole mouse brain. Nat Methods 4: 331-336.

Feng G, Mellor RH, Bernstein M, Keller-Peck C, Nguyen QT, Wallace M, Nerbonne JM, Lichtman JW, Sanes JR. 2000. Imaging neuronal subsets in transgenic mice expressing multiple spectral variants of GFP. Neuron 28: 41-51.

Helmchen F, Denk W. 2005. Deep tissue two-photon microscopy. Nat Methods 2: 932-940.

Huisken J, Stainier DY. 2009. Selective plane illumination microscopy techniques in developmental biology. Development 136: 19631975.
Huisken J, Swoger J, del Bene F, Wittbrodt J, Stelzer EH. 2004. Optical sectioning deep inside live embryos by selective plane illumination microscopy. Science 305: 1007-1009.

Jährling N, Becker K, Kramer ER, Dodt HU. 2008. 3D-visualization of nerve fiber bundles by ultramicroscopy. Med Laser Appl 23: 209-215.

Jährling N, Becker K, Dodt HU. 2009. 3D-reconstruction of blood vessels by ultramicroscopy. Organogenesis 5: 50-54.

Jährling N, Becker K, Schönbauer C, Schnorrer F, Dodt HU. 2010. Threedimensional reconstruction and segmentation of intact Drosophila by ultramicroscopy. Front Syst Neurosci 4: 1-6.

Sasnett MW, Johnston TF Jr. 1991. Beam characterization and measurement of propagation attributes. Proc SPIE 1414: 21-32.

Shaw PJ. 1995. Comparison of wide-field/deconvolution and confocal microscopy for 3D imaging. In Handbook of biological confocal microscopy, 2nd ed. (ed. Pawley JB), pp. 373-387. Plenum, New York.

Siedentopf H, Zsigmondy R. 1903. Visualization and size measurement of ultramicroscopic particles, with special application to gold-colored ruby glass. Ann Phys 10: 1-39.

Siegman AE. 1986. Wave optics and Gaussian beams, and physical properties of Gaussian beams. In Lasers, pp. 626-662 and 663-697. University Science Books, Sausalito, CA.

Siegman AE. 1998. How to (maybe) measure laser beam quality. In Diode pumped solid state (DPSS) lasers: Applications and issues (OSA Trends in Optics and Phottonics series) (ed. Dowley MW), Vol. 17. Optical Society of America, Washington, DC.

Spalteholz W. 1914. Über das Durchsichtigmachen von menschlichen und tierischen Präparaten S. Hierzel, Leipzig, Germany.

Tyszka JM, Fraser SE, Jacobs RE. 2005. Magnetic resonance microscopy: Recent advantages and applications. Curr Opin Biotechnol 26: 93-99.

Voie AH, Burns DH, Spelman FA. 1993. Orthogonal-plane fluorescence optical sectioning: Three dimensional imaging of macroscopic biological specimens. J Microsc 170: 229-236.

Weninger WJ, Mohun T. 2002. Phenotyping transgenic embryos: A rapid 3D screening method based on episcopic fluorescence image capturing. Nat Genet 30: 59-65. 


\section{Ultramicroscopy: Light-Sheet-Based Microscopy for Imaging Centimeter-Sized Objects with Micrometer Resolution}

Klaus Becker, Nina Jährling, Saiedeh Saghafi and Hans-Ulrich Dodt

Cold Spring Harb Protoc; doi: 10.1101/pdb.top076539

\begin{tabular}{rc}
$\begin{array}{r}\text { Email Alerting } \\
\text { Service }\end{array}$ & Receive free email alerts when new articles cite this article - click here. \\
\hline $\begin{array}{r}\text { Subject } \\
\text { Categories }\end{array}$ & $\begin{array}{c}\text { Browse articles on similar topics from Cold Spring Harbor Protocols. } \\
\text { Imaging/Microscopy, general (579 articles) } \\
\text { Light Microscopy (61 articles) }\end{array}$ \\
\hline
\end{tabular}

